\title{
The effect of crystalloid infusion on body water content and intra-abdominal pressure in patients undergoing orthopedic surgery under spinal anesthesia
}

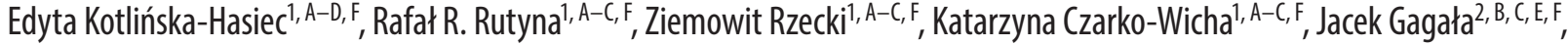

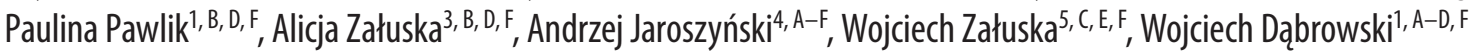 \\ ${ }^{1}$ Department of Anesthesiology and Intensive Therapy, Medical University of Lublin, Poland \\ ${ }^{2}$ Department of Orthopedic Surgery and Traumatology, Medical University of Lublin, Poland \\ ${ }^{3}$ Department of Physiotherapy, Medical University of Lublin, Poland \\ ${ }^{4}$ Department of Family Medicine, Medical University of Lublin, Poland \\ ${ }^{5}$ Department of Nephrology, Medical University of Lublin, Poland \\ A - research concept and design; $\mathrm{B}$ - collection and/or assembly of data; $\mathrm{C}$ - data analysis and interpretation; \\ $D$ - writing the article; $E$ - critical revision of the article; $F$ - final approval of the article
}

\section{Address for correspondence}

Wojciech Dąbrowski

E-mail:w.dabrowski5@yahoo.com

Funding sources

None declared

Conflict of interest

None declared

Received on November 26, 2015

Reviewed on March 7, 2016

Accepted on May 12, 2016

\begin{abstract}
Background. Crystalloids are frequently used for the correction of spinal anesthesia-induced hypotension, intraoperative bleeding, or vaporisation from surgical wounds.

Objectives. The aim of this study was to observe the effect of perioperative crystalloid infusion on intraabdominal pressure (IAP), volume excess (VE), total body water (TBW), and extracellular body water (ECW) in patients undergoing elective orthopedic surgery under spinal anesthesia.
\end{abstract}

Material and methods. Adult patients undergoing hip or knee replacement were studied. Changes in VE, TBW, ECW, and IAP were observed in patients who received restrictive fluid therapy (group R) and in patients who received liberal fluid therapy (group L). IAP was measured in the urinary bladder. All parameters were measured at 4 points in time: just before anesthesia (baseline value, A); just after surgery (B); $3 \mathrm{~h}$ after surgery (C); and on the morning of postoperative day 1 (D). Additionally, IAP was measured after anesthesia, just before surgery (A1).

Results. The mean baseline values of IAP, ECW, TBW, and VE were comparable between groups $L$ and $R$. The induction of anesthesia reduced IAP in both groups $(p<0.001)$. IAP and VE increased in both groups after surgery. Significantly higher values of IAP, however, were noted in group $L$ at time points $B, C$, and $D$. TBW and ECW increased after surgery in group $L$. In group $R, E C W$ slightly increased only at time point $C$. IAP strongly correlated with $E C W$ in group $L(p<0.001, r=0.62)$.

Conclusions. Spinal anesthesia reduces IAP. A perioperative increase in body water content and IAP mainly depends on the volume of the infused crystalloids.

Key words: fluid therapy, intra-abdominal pressure, extracellular water content, volume excess, liberal vs restricted

DOI

10.17219/acem/63140

Copyright

Copyright by Author(s)

This is an article distributed under the terms of the

Creative Commons Attribution Non-Commercial License

(http://creativecommons.org/licenses/by-nc-nd/4.0/) 
Bioimpedance is a popular non-invasive, safe, and relatively cheap technique, which provides useful information in the case of patients with chronic kidney diseases, acute kidney injury, or nutritional disorders. ${ }^{1,2}$ Bioimpedance is considered to be a sensitive tool for measuring body fluid composition; however, the interpretation of this data is difficult. The raw impedance data, consisting of resistance and reactance values, are abstract, and provide only indirect information on extraand intracellular fluid volumes. In practical applications, this problem has been solved by whole-body bioimpedance (BIA) measurements, which analyze the bioelectrical properties between the wrist and the ankle, assuming a steady fluid distribution. The volume of total body water (TBW), extracellular body water (ECW), intracellular body water (ICW), and volume excess (VE) can be quickly and reliably estimated using BIA. ${ }^{3,4}$ Several studies have documented significant benefits resulting from BIA-controlled fluid balance. ${ }^{3,5,6}$ Unfortunately, only 1 study has analysed the effect of perioperative fluid therapy on body water distribution in patients undergoing various elective gynecological procedures under general anesthesia. ${ }^{6}$ In addition, the effect of crystalloid therapy has not been documented in conscious spinal-anesthetized patients.

Crystalloid infusion is routinely used for the correction of anesthesia-related hemodynamic disorders. Clinical assessment of fluid status is subjective and frequently based on clinical experience, and the current clinical practice for fluid administration remains controversial. A rapid decrease in blood pressure after the induction of spinal anesthesia implies dehydration and causes fluid infusion, which results in a postoperative positive fluid balance. Such hypervolemia is needed to preserve organ perfusion during regional anesthesia. ${ }^{7}$ Unfortunately, positive perioperative fluid balance leading to tissue edema following a surgery-related increase in vascular permeability is associated with severe postoperative complications. $^{7-9}$ The adverse effects of fluid overload are most evident in the lungs and gastrointestinal tract. Fluid overload leads to pulmonary edema, which may decrease gas exchange, and makes the patients more susceptible to infections. Moreover, VE connected with surgeryrelated inflammatory response results in interstitial edema, increasing intra-abdominal pressure (IAP)..$^{7-10}$ Regrettably, the adjustment and control of fluid overload is difficult and BIA seems to be a more user-friendly method for managing perioperative fluid therapy.

The purpose of this study was to observe the effect of perioperative crystalloid infusion on IAP and body water content in patients undergoing elective orthopedic surgery under spinal anesthesia.

\section{Material and methods}

\section{Ethics statement}

This prospective observational study was conducted in adult patients undergoing elective orthopedic surgery under spinal anesthesia. The study was conducted in accordance with the Declaration of Helsinki, and was approved by the Institutional Review Board and the Bioethical Committee for Human Studies of the Medical University of Lublin, Poland (KE-0254/58/2010). Written informed consent was obtained from all participants.

\section{Anesthesia and surgery}

Patients were clinically assessed 1 day before surgery, and only patients with ASA I or II (American Society of Anesthesiologists, www.asahq.org)were selected for the present study. ${ }^{11}$ Patients with heart disease or severe respiratory diseases, or those receiving medication that alters the physiological response to fluid infusion (such as $\beta$-blockers, angiotensin converting enzyme inhibitors, or digoxin) were excluded. Moreover, patients with chronic renal insufficiency, with a history of severe abdominal diseases or alcoholism, and those who required massive intra-operative blood transfusion or perioperative massive fluid resuscitation were also excluded.

A preoperative routine blood examination was carried out to assess complete blood count, blood glucose, urea, creatinine and electrolyte levels $\left(\mathrm{K}^{+}, \mathrm{Na}^{+}\right)$, and blood group. Additionally, chest X-ray and electrocardiogram were done prior to surgery. The day before surgery all patients received a single oral dose (2 $\mathrm{mg}$ ) of estazolam (Estazolam, Polfa Tarachomin, Poland). Before the induction of anesthesia, patients were routinely monitored for continuous electrocardiography and pulse oximetry $\left(\mathrm{SpO}_{2}\right)$, and arterial blood pressure was measured intermittently. An 18- or 17-gauge intravenous cannula was inserted into the peripheral vein. The urinary bladder was catheterized in all patients before anesthesia induction.

Before the induction of anesthesia, all patients were routinely monitored with respect to electrocardiography (leads II or III), arterial blood pressure and peripheral blood saturation (Infinity Delta, Dreager Medical System, Telford, USA). The induction of spinal anesthesia was performed in the lateral position, at the L2-L3 or L3-L4 level. After skin sanitization, a 26-gauge pencilpoint needle (Balton, Warsaw, Poland) or a 27-gauge needle (Pencan ${ }^{\circledR}$, Braun, Melsungen, Germany) was used for spinal space identification. The induction of anesthesia was performed with $0.5 \%$ bupivacaine hydrochloride (Marcaine Spinal, AstraZeneca AB, Stockholm, Sweden) in a single dose depending on the patient's height, weight, and physical condition (doses of 15-20 mg). The level of spinal anesthesia was confirmed 5 min after the anesthetic injection in the supine position.

Systolic and diastolic blood pressures were measured at 3-5 min intervals. Hypotension was defined as a fall in systolic blood pressure of $25 \%$ of the baseline value, or an absolute value lower than $90 \mathrm{~mm} \mathrm{Hg}$. All patients received an infusion of the balanced electrolyte crystalloids (PWE or Ringer, Polfa, Kutno, Poland). Changes in IAP, VE, 
TBW, and ECW were observed in patients who received liberal fluid therapy at a dose of $30 \mathrm{~mL}$ of crystalloids per $\mathrm{kg}$ of body weight/h intraoperatively and $5 \mathrm{~mL}$ of crystalloids per $\mathrm{kg}$ of body weight in the first 3 postoperative hours (group L), and in patients who received $10 \mathrm{~mL}$ of crystalloids per $\mathrm{kg}$ of body weight/h intraoperatively and $5 \mathrm{~mL}$ of crystalloids per $\mathrm{kg}$ body weight during the first 3 postoperative hours (group R). ${ }^{12}$ In group L, spinal-related hypotension was treated with a rapid infusion of 1,000-1,500 $\mathrm{mL}$, whereas in group $\mathrm{R}$, spinal-related hypotension was treated with a maximum of $500 \mathrm{~mL}$ of balanced electrolyte crystalloids and an ephedrine hydrochloride (Ephedrine, Polfa, Warsaw, Poland) at a dosage of 5-25 mg intravenously. In group L, a single dose (5-10 mg) of ephedrine hydrochloride was given to patients who did not respond to fluid administration higher than $1,500 \mathrm{~mL}$. The randomization was performed using a concealed envelope method. All participants were assessed clinically in case of any postoperative complications or hemodynamic instability. On the morning of postoperative day 1, a control blood examination was carried out to assess complete blood count, blood glucose, urea, creatinine, and electrolyte levels.

\section{Data collection and study protocol}

IAP was measured in the urinary bladder after an injection of $25 \mathrm{~mL}$ of sterile saline, in the complete supine position with the transducer zeroed at the level of the midaxillary line (Kron technique). VE, TBW, and ECW were measured by whole-body BIA using the Body Composition Monitor (BCM, Fresenius Medical Care, Hamburg, Germany). Prior to examination, the height and body mass of the patient were measured. Electrodes (Fresenius Medical Care, Hamburg, Germany) were placed on the wrist (proximally to the metacarpophalangeal joint) and ankle (proximally to the transverse metatarsal arch on the superior side of the foot). Bioimpedance was measured at 50 frequencies ranging from $5 \mathrm{kHz}$ to $1 \mathrm{MHz}$ in the supine body position. In all patients, the electrodes were placed on the body side rather than on the surgery side in order to avoid the effects of local surgery-induced tissue edema.

All parameters were measured at the following 4 points in time: just before surgery (A); just after surgery (B); $3 \mathrm{~h}$ after surgery $(\mathrm{C})$; and on the morning of postoperative day 1 (D). Additionally, IAP was measured just before surgery, after the induction of spinal anesthesia (time point A1).

\section{Statistics}

The data was analyzed using STATISTICS v. 9.0.0 software (IBM, Chicago, USA). Initially, the normality of the distribution of variables was analyzed by the Shapiro-Wilk test. Means and standard deviations (SD) were calculated for normally distributed data, and Student's unpaired t-test and Pearson's $\chi^{2}$ test were used to compare the variables. Non-parametric data were statistically analyzed using the ANOVA Friedman test and the post hoc Dunn's test with Bonferroni correction. The ANOVA KruskalWallis test and the post hoc Dunn's test with Bonferroni correction was used for the initial detection of differences between groups L and R. The Spearman's rank correlation test was used for the overall analysis. The pilot data was collected form patients receiving liberal fluid therapy for the calculation of a minimal sample. The minimal sample of participants was calculated for differences in IAP and VE between time points A and B. The power of all statistical tests was determined by $G$ *Power software (1- $\beta$ ).

\section{Results}

Seventy adult patients ( 28 females and 42 males) aged 19-85 undergoing elective orthopedic surgery were enrolled into the present study. There were no differences in demographic parameters (age, sex, height, weight, and BMI) between groups L and R. In group L, 2 patients were excluded due to significant intraoperative bleeding and 1 withdrew his consent for the study immediately after surgery. In group R, 4 patients were excluded due to the need for massive fluid and blood resuscitation during surgery and/or the early postoperative period. Finally, 63 patients were studied: 32 of them were assigned to group L (13 females aged 35-82, and 19 males aged 19-78) and 31 of them were assigned to group $\mathrm{R}$ (11 females aged 39-85, and 20 males aged 35-79). In group L, 27 patients underwent hip replacement and 5 were subjected to knee arthroplasty or arthroscopic surgery, whereas in group $\mathrm{R}$, 28 patients underwent hip replacement and 3 had knee surgery $\left(\chi^{2}=0.5\right.$ for $\mathrm{p}=0.478$, and $\chi^{2}$ with Yates correction $=0.11$ for $\mathrm{p}=0.741$ ).

The demographic data, perioperative blood parameters, and hemodynamic parameters were comparable in both groups (Table 1). A resumption of normal diet and the length of stay in hospital were similar in both groups. In group L, patients received crystalloid infusion at these mean dosages: $2822 \pm 606 \mathrm{~mL}$ during surgery, $731 \pm 288 \mathrm{~mL}$ during the first 3 postoperative hours, and $1478 \pm 797 \mathrm{~mL}$ from the $3^{\text {rd }}$ postoperative hour till the morning of postoperative day 1 . The mean fluid balances were $2312 \pm 660 \mathrm{~mL}$ postoperatively, $247 \pm 334 \mathrm{~mL}$ after $3 \mathrm{~h}$, and $291 \pm 806 \mathrm{~mL}$ from the $3^{\text {rd }}$ postoperative hour till the morning of postoperative day 1 . In group $\mathrm{R}$, patients received crystalloid infusion at these mean dosages: $823 \pm 223 \mathrm{~mL}$ during surgery, $658 \pm 222 \mathrm{~mL}$ during the first 3 postoperative hours, and $583 \pm 186 \mathrm{~mL}$ from the $3^{\text {rd }}$ postoperative hour till the morning of postoperative day 1 . The mean fluid balances were $182 \pm 270 \mathrm{~mL}$ postoperatively, $373 \pm 274 \mathrm{~mL}$ after $3 \mathrm{~h}$, and $304 \pm 342 \mathrm{~mL}$ from the $3^{\text {rd }}$ postoperative hour till the morning of postoperative day 1 . Fluid balance was significantly higher just after surgery $(\mathrm{p}<0.001)$ and on the morning of postoperative day $1(\mathrm{p}<0.001)$ in patients who had received liberal crystalloid therapy. 
Table 1. Demographic data and perioperative blood and hemodynamic parameters in patients who received liberal crystalloids therapy (group L) and restrictive crystalloids therapy (group R)

\begin{tabular}{|c|c|c|c|c|}
\hline \multicolumn{2}{|r|}{ Parameter } & Group L & Group R & $\mathrm{p}$-value \\
\hline \multirow{5}{*}{ 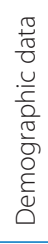 } & Baseline weight (kg) & $80.99 \pm 12.27$ & $78.4 \pm 18.06$ & 0.895 \\
\hline & BMI $\left(\mathrm{kg} / \mathrm{m}^{2}\right)$ & $27.22 \pm 4.00$ & $26.95 \pm 4.58$ & 0.076 \\
\hline & $\mathrm{BSA}\left(\mathrm{m}^{2}\right)$ & $2.02 \pm 0.15$ & $1.91 \pm 0.27$ & 0.279 \\
\hline & Anesthesia (min) & $112 \pm 22$ & $104 \pm 46$ & 0.846 \\
\hline & Surgery (min) & $88 \pm 18$ & $79 \pm 35$ & 0.46 \\
\hline \multirow{10}{*}{ 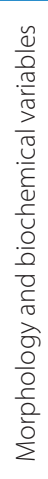 } & Preoperative $\mathrm{Hb}$ (g\%) & $12.2 \pm 1.27$ & $12.5 \pm 1.42$ & 0.743 \\
\hline & Postoperative $\mathrm{Hb}(\mathrm{g} \%)$ & $9.91 \pm 1.95$ & $10.34 \pm 1.92$ & 0.341 \\
\hline & Preoperative Ht (\%) & $34.4 \pm 2.67$ & $33.9 \pm 2.95$ & 0.621 \\
\hline & Postoperative Ht (\%) & $28.9 \pm 2.65$ & $30.2 \pm 2.82$ & 0.094 \\
\hline & Preoperative blood glucose (mg\%) & $98.6 \pm 10.3$ & $96.5 \pm 9.73$ & 0.893 \\
\hline & Postoperative blood glucose (mg\%) & $124.5 \pm 21.6$ & $132.2 \pm 21.7$ & 0.237 \\
\hline & Preoperative serum creatinine $(\mathrm{mg} / \mathrm{dL})$ & $0.8 \pm 0.25$ & $0.86 \pm 0.18$ & 0.898 \\
\hline & Postoperative serum creatinine $(\mathrm{mg} / \mathrm{dL})$ & $0.97 \pm 0.21$ & $1.1 \pm 0.72$ & 0.754 \\
\hline & Preoperative serum urea (mg/dL) & $32.3 \pm 7.5$ & $33.7 \pm 8.3$ & 0.542 \\
\hline & Postoperative serum urea (mg/dL) & $45.3 \pm 10.6$ & $48.4 \pm 14.5$ & 0.078 \\
\hline
\end{tabular}

There were no differences in blood account, creatinine level, serum urine and blood glucose concentration in group $L$ and $R$ (Student's t-test).

Table 2. Changes in volume excess (VE) in patients who received liberal crystalloids therapy (group L) and restrictive crystalloids therapy (group R) - median (quartile 1 and 3)

\begin{tabular}{c|c|c|c|c|}
\multirow{2}{*}{$\begin{array}{c}\text { Volume } \\
\text { excess (L) }\end{array}$} & \multicolumn{4}{|c|}{ Time points } \\
\cline { 2 - 5 } & $\mathrm{A}$ & $\mathrm{B}$ & $\mathrm{C}$ & $\mathrm{D}$ \\
\hline \multirow{2}{*}{ Group L } & -0.47 & $0.93^{* * * \neq \neq}$ & $0.63^{* * * \neq \neq}$ & $0.35^{* *}$ \\
& $(-1.6,0)$ & $(-0.28,1.9)$ & $(0.25,1.95)$ & $(-0.21,2.5)$ \\
Group R & -1.1 & $0^{* *}$ & $0.1^{* * *}$ & $0.2^{* * *}$ \\
& $(-2.1,0.2)$ & $(-0.9,0.7)$ & $(-0.4,0.5)$ & $(-0.2,0.9)$ \\
\hline
\end{tabular}

Time points: A - just before anesthesia induction; B - just after surgery; $\mathrm{C}-3 \mathrm{~h}$ after surgery; and $\mathrm{D}$ - on the morning of postoperative day 1 ; ${ }^{* *} p<0.01$ and ${ }^{* * *} p<0.001$ - significant differences with baseline value (ANOVA Friedman test and post hoc Dunn's test with Bonferroni correction);

${ }^{\ddagger \ddagger} p<0.01$ - significant differences between groups $L$ and R (ANOVA

Kruskall-Wallis test and post hoc Dunn's testwith Bonferroni correction).

Table 3. Changes in total body water (TBW) and extracellular body water (ECW) content in patients who received liberal crystalloids therapy (group L) and restrictive crystalloids therapy (group R) - median (quartile 1 and 3)

\begin{tabular}{|c|c|c|c|c|c|}
\hline \multirow{2}{*}{\multicolumn{2}{|c|}{$\begin{array}{c}\text { BCM } \\
\text { variables }\end{array}$}} & \multicolumn{4}{|c|}{ Time points } \\
\hline & & $\mathrm{A}$ & B & C & D \\
\hline \multirow{2}{*}{ 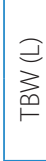 } & Group L & $\begin{array}{c}37.25 \\
(34.55,42.93)\end{array}$ & $\begin{array}{c}39.7^{* * *} \\
(36.79,48.79)\end{array}$ & $\begin{array}{c}41.17^{* * *} \\
(37.46,46.5)\end{array}$ & $\begin{array}{c}43.37^{* * *} \\
(37.41,48.2)\end{array}$ \\
\hline & Group R & $\begin{array}{c}44.3 \\
(39.7,48.8)\end{array}$ & $\begin{array}{c}44.5 \\
(37.2,50.9)\end{array}$ & $\begin{array}{c}43.6 \\
(37.9,48.8)\end{array}$ & $\begin{array}{c}43.5 \\
(35.1,51.2)\end{array}$ \\
\hline \multirow{2}{*}{$\stackrel{Ð}{\circlearrowright}$} & Group L & $\begin{array}{c}18.7 \\
(16.7,20.4)\end{array}$ & $\begin{array}{c}20^{* * * \neq} \\
(17.7,22.7)\end{array}$ & $\begin{array}{c}21.6^{* * * * \neq} \\
(18.3,22.8)\end{array}$ & $\begin{array}{c}21.2^{* * * *} \\
(17.6,23.2)\end{array}$ \\
\hline & Group R & $\begin{array}{c}17 \\
(13,20.5)\end{array}$ & $\begin{array}{c}18 \\
(13.5,20.4)\end{array}$ & $\begin{array}{c}18.5 \\
(13.9,20.3)\end{array}$ & $\begin{array}{c}19.2^{*} \\
(13.9,19.9)\end{array}$ \\
\hline
\end{tabular}

Time points: A - just before anesthesia induction; B - just after surgery; $C$ - $3 \mathrm{~h}$ after surgery; and $D$ - on the morning of postoperative day $1 ;{ }^{*} \mathrm{p}<0.05$, ${ }^{* *}$ $p<0.01$ and ${ }^{* * *} p<0.001$ - significant differences with baseline value (ANOVA Friedman test and post hoc Dunn's test with Bonferroni correction); ${ }^{\ddagger} p<0.05$ and ${ }^{\ddagger \neq} p<0.01$ - significant differences between groups $L$ and $R$ (ANOVA Kruskall-Wallis test and post hoc Dunn's test with Bonferroni correction).
Power calculations suggested that only 22 patients would be needed to get a credible difference in IAP measurement at time points $A$ and $B(1-\beta=0.96$ for $\mathrm{p}<0.05)$. The mean baseline values of IAP were comparable in groups $\mathrm{L}$ and $\mathrm{R}(3.94 \pm 1.68 \mathrm{~mm} \mathrm{Hg}$ and $3.69 \pm 2.09 \mathrm{~mm} \mathrm{Hg}$, respectively). The induction of spinal anesthesia reduced IAP in both groups. IAP increased in both groups after surgery; significantly higher values of IAP, however, were noted in group $\mathrm{L}$ just after surgery, $3 \mathrm{~h}$ after surgery, and on the morning of postoperative day 1 (Fig. 1). In all patients, IAP was lower than $12 \mathrm{~mm}$ $\mathrm{Hg}$, though a value of $11 \mathrm{~mm} \mathrm{Hg}$ was noted in 3 patients from group L just after surgery and $3 \mathrm{~h}$ after surgery.

Power calculations suggested that only 32 patients would be needed to get a credible difference in VE measurement at time points $A$ and $B(1-\beta=0.95$ for $p<0.05)$. The baseline value of VE was comparable in both groups (Table 2). Crystalloid infusion increased VE in both groups, but liberal crystalloid infusion increased VE by significantly more than did restrictive infusion. Significantly higher values of VE were noted in both groups during the early postoperative period (Table 2).

The baseline values of TBW and ECW were comparable in both groups. Liberal crystalloid infusion increased TBW, whereas restrictive infusion did not change TBW (Table 3). There were no differences between groups L and $R$ in TBW. A significantly higher value of ECW was noted in patients that received liberal crystalloid therapy. ECW increased just after surgery, $3 \mathrm{~h}$ after surgery, and on the morning of postoperative day 1. Restrictive crystalloid therapy elevated ECW only $3 \mathrm{~h}$ after surgery (Table 3). Higher ECW was noted in Group L just after surgery and on the morning of postoperative day 1 .

In all studied patients, IAP correlated with ECW $(\mathrm{p}<0.001, \mathrm{r}=0.57)$. In patients who received restrictive crystalloid therapy, IAP slightly correlated with ECW ( $\mathrm{p}<0.001, \mathrm{r}=0.47$ ), whereas in patients who received liberal crystalloid therapy, IAP strongly correlated with ECW (Fig. 2), as well as with TBW (p $<0.001, \mathrm{r}=0.44$ ) and VE $(\mathrm{p}<0.01, \mathrm{r}=0.29)$.

\section{Discussion}

We have documented here the adverse effect of crystalloid infusion on IAP and body water content in patients undergoing elective orthopedic surgery. Liberal crystalloid 
Fig. 1. Changes in intra-abdominal pressure in patients who received liberal fluid therapy (窓 - group L) and restrictive fluid therapy ( - group R) during surgery

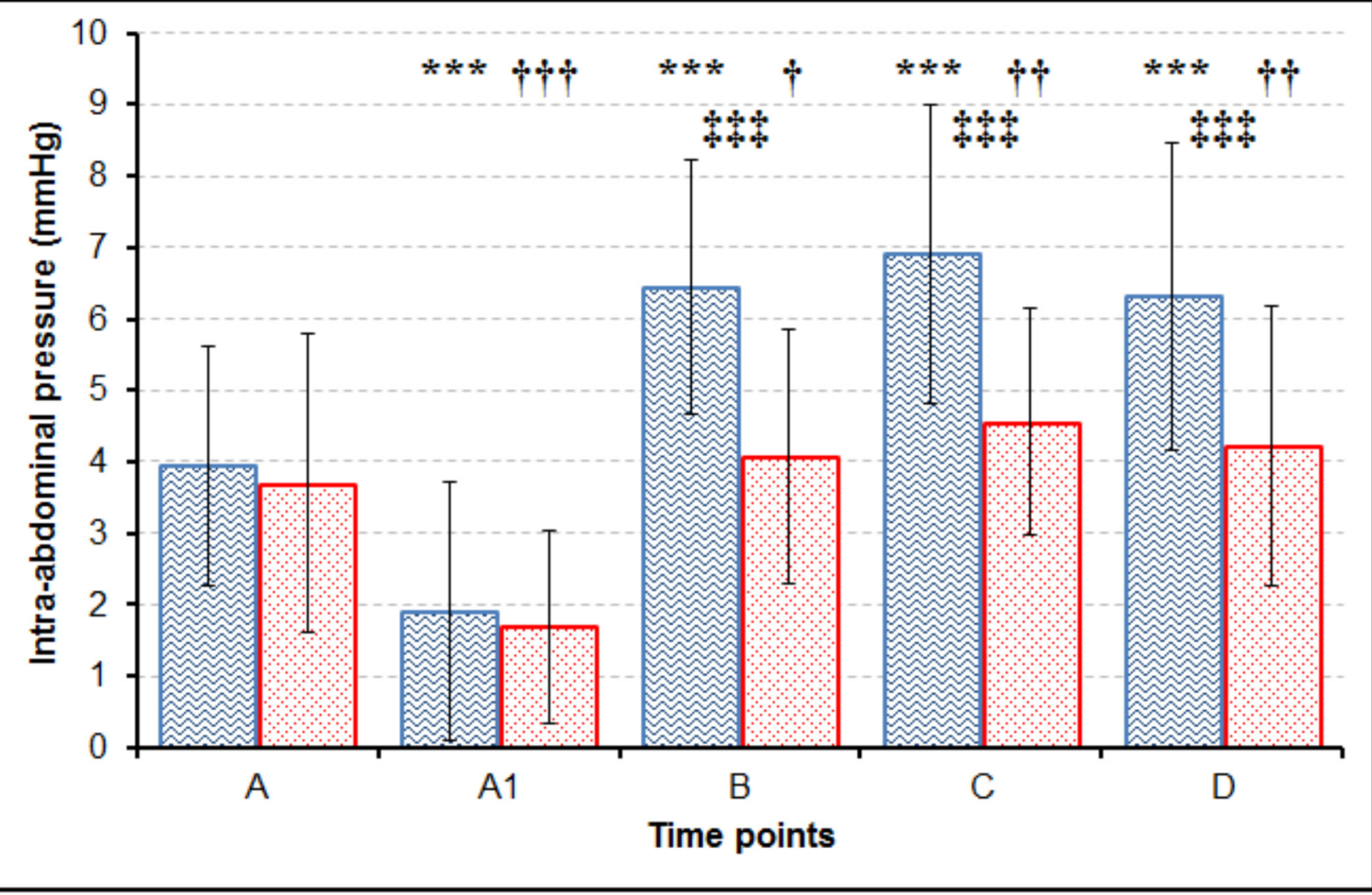

Time points: A - just before anesthesia induction; A1 - after anesthesia induction, just before surgery; B - just after surgery; $\mathrm{C}-3$ hours after surgery; and $\mathrm{D}$ - on the morning of postoperative day $1 ;{ }^{* * *} \mathrm{p}<0.001$ - significant differences compared with baseline value in group $\mathrm{L} ;{ }^{\dagger} \mathrm{p}<0.05$,

${ }^{\dagger+} p<0.01$ and ${ }^{\ddagger \neq \ddagger} p<0.001$ - significant differences compared with baseline value in group $R_{;}{ }^{\ddagger \neq \neq} p<0.001$ - significant differences between groups $L$ and $R$ (Student's t-test).

infusion elevated IAP and increased VE, TBE, and ECW, whereas restrictive crystalloid infusion only slightly affected those parameters. Additionally, IAP was strongly correlated with ECW in patients who received liberal crystalloid therapy. Such a strong correlation was not observed in patients who received restrictive crystalloid infusion.

Fluid management is a fundamental component of perioperative treatment. There is increasing evidence that fluid infusion affects postoperative outcomes, and plays a crucial role in critically ill patients. ${ }^{8,9,13,14}$ Excessive perioperative fluid loading leads to extravascular fluid accumulation and weight gain, which corresponds with increased mortality and morbidity during the early postoperative period. $6,8,15$ Several meta-analyses have reported that goaloriented fluid therapy significantly reduces renal and pulmonary postoperative complications, and accelerates the first bowel movement, the resumption of normal diet, and shortens the length of stay in hospital. Restrictive fluid administration reduces postoperative pneumonia and pulmonary edema. ${ }^{6,15,16}$ An inappropriate fluid administration disturbs the liberation of atrial natriuretic peptide, and injures the endothelial glycocalix, leading to the damage of the vascular barrier and increased fluid shifts into the extravascular space., ${ }^{7,17}$ This pathology may result in abnormal extravascular water content, tissue edema, and raised IAP. The present study also shows that perioperative liberal crystalloid infusion disturbs the body water balance by increasing TBW and ECW, and that restrictive crystalloid therapy does not affect body water content. Moreover, elevated ECW corresponds with IAP, which is in line with our previous observation. ${ }^{18}$ In a recent study, we documented a strong correlation between IAP and ECW, both in critically ill patients and in patients undergoing extra-abdominal surgery. We also estimated 24.9 L of ECW and 9.5 L of VE as cut-off points for the development of intra-abdominal hypertension (IAP higher than $12 \mathrm{~mm} \mathrm{Hg}) .{ }^{18}$ In the present study, none of the participants had ECW and VE higher than the above-mentioned values, and intra-abdominal hypertension was not noted during the study, although IAP increased to $11 \mathrm{~mm} \mathrm{Hg}$ in 3 patients from the liberal crystalloid therapy group. It is worth noting that those patients received a higher volume of crystalloids (4167 $\pm 289 \mathrm{~mL}$ ), and their duration of surgery was higher than others' in group L. Based on this observation, we can speculate that a perioperative increase in IAP corresponds to the duration of surgery and the type of fluid therapy; however, this hypothesis should be confirmed by further study. 
Fig. 2. Correlation between intra-abdominal pressure (IAP) and extracellular water content (ECW) in patients undergoing liberal crystalloid therapy during orthopedic surgery and the early postoperative period ( $p<0.001, r=0.62$; Spearman's rank correlation test)

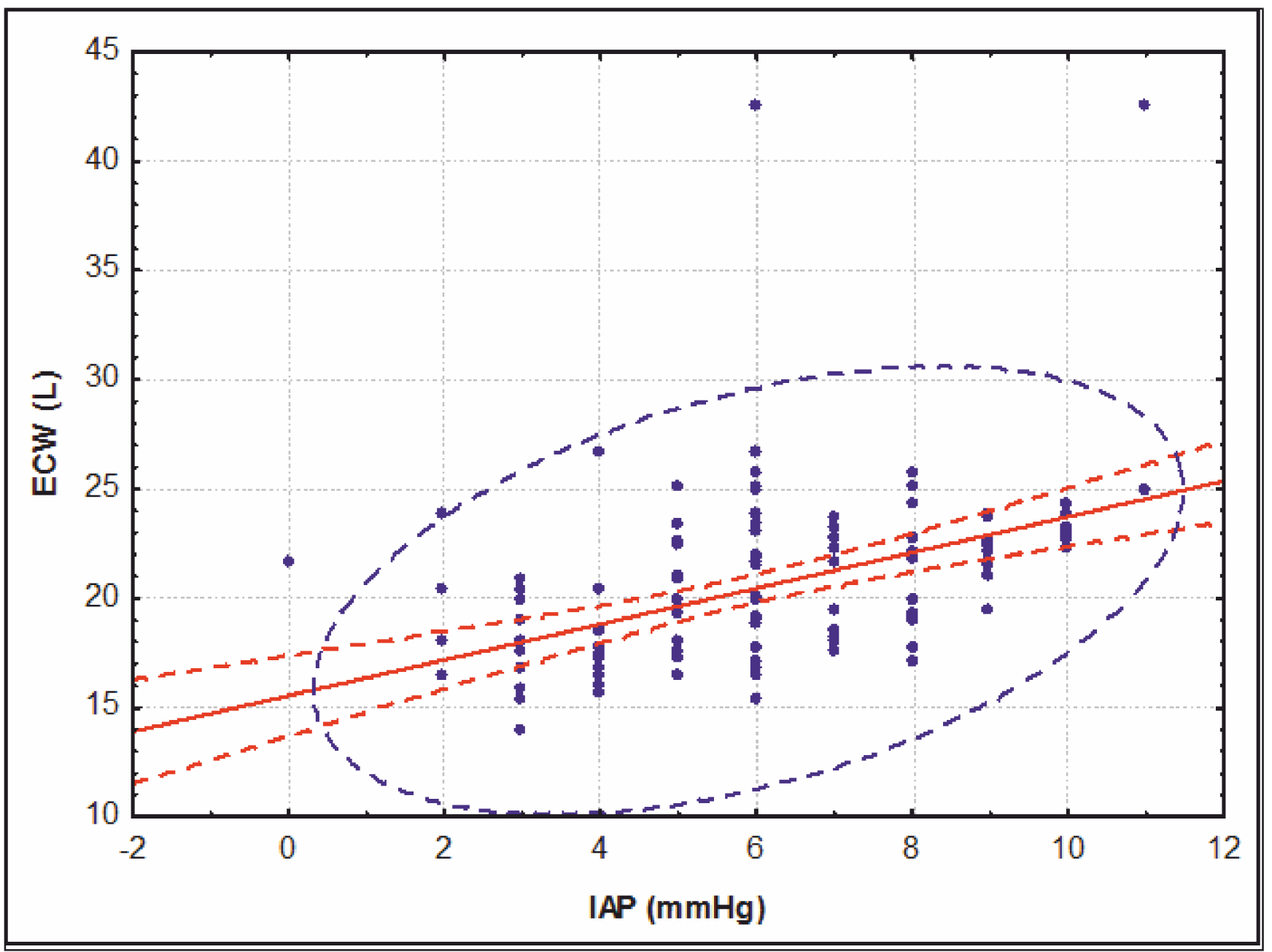

Crystalloids are electrolyte solutions which are frequently used to replace extracellular volume losses following urination, perioperative perspiration or anesthesia-related hypotension. Research from the International Fluid Optimisation Group have recommended crystalloid solutions for routine surgeries of short duration. ${ }^{19}$ Additionally, they have recommended bolus therapy rather than continuous infusion when the goal is to improve pressure, perfusion, and oxygen delivery. In the present study all patients received an initial bolus of crystalloids following anesthesia-related hypotension, though the dosages were significantly greater in patients treated with liberal fluid infusion (data not shown). We did not observe any adverse effect from intraoperative crystalloid infusion. It is worth noting that some authors observed hypercoagulation following crystalloid infusion. ${ }^{20,21}$ A rapid infusion of crystalloids increases coagulative activity following hemodilution in healthy volunteers. ${ }^{20}$ In vitro studies also document an increase in hypercoagulation measured by thrombelastography following different types of crystalloids. ${ }^{21,22}$ Interestingly, this effect seems to depend on blood magnesium concentration. ${ }^{20}$ Ruttmann et al. showed that a rapid infusion of crystalloids decreased serum magnesium concentration to the lower limits of norms and increased coagulation in healthy volunteers, when magnesium supplementation attenuated these disturbances. ${ }^{20}$ Significant hypercoagulation has also been reported in patients receiving liberal perioperative fluid therapy 24 and $48 \mathrm{~h}$ after surgery. ${ }^{12}$ This pathology has not been observed in patients receiving restrictive fluid therapy. Crystalloid-related hypercoagulation increased the frequency of deep venous thrombosis in patients receiving normal saline solutions during major abdominal surgery compared with those who did not receive intravenous fluids. ${ }^{23}$ Hip replacement and knee arthroplasty are moderately complex surgical procedures. Additionally, all of our patients routinely received low molecular heparin during the early postoperative period, and we did not note any clinical thromboembolic complications.

A postoperative increase in IAP and ECW may result from a surgery-related general inflammatory response. Indeed, a growing data set presents an increase in IAP following inflammatory-related intestine edema., 24,25 Surgery-related injury induces the release of proinflam- 
matory cytokines, such as tumor necrosis factor $\alpha$ (TNF $\alpha$ ), vascular endothelial growth factor (VEGF), many interleukins, histamine, and E-selectine, which may disturb vascular permeability, and increase fluid shift into the extravascular space. ${ }^{26,27}$ Moreover, surgery-related perioperative stress induces the release of antidiuretic hormone, cortisol and corticotrophins, and stimulates the renin-angiotensin-aldosterone system to increase salt and fluid retention. ${ }^{28,29}$ Therefore, a surgery-induced increase in vascular permeability and hormone-dependent fluid retention provoke perioperative tissue edema independently of fluid infusion. In the present study, an increase in VE just after surgery and $3 \mathrm{~h}$ after surgery may confirm this pathology. A higher increase in VE in patients who received liberal crystalloid infusion demonstrates an unfavourable effect of liberal fluid administration in patients undergoing elective surgery. Liberal crystalloid infusion also increased ECW by more than restrictive infusion. An increase in ECW following intraoperative fluid infusion has also been observed by Ernstbrunner et al. ${ }^{5}$ They observed a strong correlation between the net perioperative fluid balance and a change in ECW in patients undergoing elective gynecological surgery under general anesthesia. This increase in ECW corresponded to TBW, but the authors proposed net perioperative fluid balance and postoperative capillary leak index as significant individual predictors for changes in ECW. Thus, an appropriate fluid therapy associated with an increased capillary leak index may elevate ECW and subsequently IAP.

BIA is a practical tool for measuring body fluid components, and is easily applied to everyday practice. The clinical interpretation of the data may be difficult and the estimation of volume deficits and VE may pose a serious problem for the physician. It is essential to recognize that the same amount of extracellular fluid leads to different changes in whole-body bioimpedance, depending on whether this fluid is added to or removed from the leg, the arm, or the trunk. Generally, BIA has been suggested as a much more sensitive measurement of volume changes in the limbs than in the trunk. ${ }^{30}$ Other authors have proposed BIA as a sensitive and easy method for measuring perioperative fluid administration in patients undergoing elective surgery. ${ }^{5}$ We have also documented the usefulness of BIA in perioperative clinical practice, though we placed the electrodes on the body side opposite to the surgery. Surgery-related tissue edema and wound hematoma may have significantly disturbed the final results of BIA measurement. Although our measurement was performed on the body site opposite to the surgery, the BIA findings may have also corresponded to intraoperative body position. Many orthopedic surgeries are performed in the lateral position; $87 \%$ of surgeries in the present study were performed in the lateral position, with the patients laid on the measurement side. Despite a short duration of surgery, intraoperative position might have had an impact on postoperative BIA findings. Several authors have documented an unequal distribution of body water in various body positions. ${ }^{31,32}$ The corrected BIA measurement should be performed in the supine position. Extracellular volume remains constant after a change of body position from supine to standing, whereas BIA findings present an apparent increase in ECW. This effect may result from a higher sensitivity to fluid administration in the limbs than in the trunk. ${ }^{32}$ Similar effects have been observed during hemodialysis and ultrafiltration. ${ }^{33}$ In the present study, all BIA measurements were performed in the supine position. However, we could speculate that a lateral position during surgery might disturb body water distribution, thus affecting measurement just after surgery. Therefore, this study should be continued in patients operated on in the supine position.

Finally, we demonstrate the effect of different types of perioperative crystalloid therapy on body water distribution in patients undergoing elective orthopedic surgery. Liberal fluid infusion significantly affects body water content, especially ECW, VE, and TBW, whereas restrictive crystalloid infusion does not affect body water content. Moreover, liberal crystalloid infusion increases IAP by significantly more than restrictive infusion. We also show that IAP is correlated with ECW. Based on our findings, we suggest that the observation of changes in IAP following fluid resuscitation can provide valuable information on extracellular water content.

\section{References}

1. Onofriescu M, Hogas $S$, Voroneanu $L$, et al. Bioimpedance-guided fluid management in maintenance hemodialysis: A pilot randomized controlled trial. Am J Kidney Dis. 2014;64:111-118.

2. Dąbrowski W, Kotlińska-Hasiec E, Schneditz D, et al. Continuous veno-venous hemofiltration to adjust fluid volume excess in septic shock patients reduces intra-abdominal pressure. Clin Nephrol. 2014;82:41-50.

3. Zaluska WT, Schneditz D, Kaufman AM, Morris AT, Levin NW. Relative underestimation of fluid removal during hemodialysis hypotension measured by whole body bioimpedance. ASAIO J. 1998;44:823-27.

4. Malbrain MLNG, Huygh J, Dąbrowski W, De Waele JJ, Staelens A, Wauters J. The use of bio-electrical impedance analysis (BIA) to guide fluid management, resuscitation and deresuscitation in critically ill patients: A bench-to-bedside review. Anesthesiol Intensive Ther. 2014;46:381-91.

5. Ernstbrunner M, Kostner L, Kimberger O, et al. Bioimpedance spectroscopy for assessment of volume status in patients before and after general anesthesia. PLoS One. 2014;9:e111139. doi: 10.1371/ journal.pone.0111139.

6. Silva JM Jr, de Oliveira AM, Nogueira FA, et al. The effect of excess fluid balance on the mortality rate of surgical patients: A multicenter prospective study. Crit Care. 2013;17:R288. doi: 10.1186/cc13151.

7. Hahn RG. Must hypervolaemia be avoided? A critique of the evidence. Anaesthesiol Intensive Ther. 2015;47:449-456.

8. Berger MM, Gradwohl I, Brunauer A, Ulmer H, Dünser MW. Targets of perioperative fluid therapy and their effects on postoperative outcome: A systematic review and meta-analysis. Minerva Anestesiol. 2015;81:794-807.

9. Elvevoll B, Husby $\mathrm{P}, \varnothing \mathrm{vrebø} \mathrm{K,} \mathrm{Haugen} \mathrm{O.} \mathrm{Acute} \mathrm{elevation} \mathrm{of} \mathrm{intra-}$ abdominal pressure contributes to extravascular shift of fluid and proteins in an experimental porcine model. BMC Research Notes. 2014;7:738. 
10. Kraemer M, Rode C, Wizemann V. Detection limit of methods to assess fluid status changes in dialysis patients. Kidney Int. 2006;69:1609-1620.

11. Dripps RD, Lamont A, Eckenhoff JE. The role of anesthesia in surgical mortality. J Am Med Assoc. 1961;178:261-266.

12. Holte K, Kristensen BB, Valentiner L, Foss NB, Husted H, Kehlet H. Liberal versus restrictive fluid management in knee arthroplasty: A randomized, double-blind study. Anesth Analg. 2007;105:465-474.

13. Jiang $L$, Jiang $S$, Zhang $M$, Zheng $Z$, Ma Y. Albumin versus other fluids for fluid resuscitation in patients with sepsis: A meta-analysis. PLoS One. 2014;9(12):e114666. doi:10.1371/journal.pone.0114666

14. Simon TP, Thiele C, Schuerholz T, et al. Molecular weight and molar substitution are more important in HES-induced renal impairment than concentration after hemorrhagic and septic shock. Minerva Anestesiol. 2015;81:608-618.

15. Cohen R. Colloids versus crystalloids: The story continues. Minerva Anestesiol. 2013;79:3-4.

16. Corcoran T, Rhodes JE, Clarke S, Myles PS, Ho KM. Perioperative fluid management strategies in major surgery: A stratified metaanalysis. Anesth Analg. 2012;114:640-651.

17. Bruegger $D$, Jacob $M$, Rehm $M$, et al. Atrial natriuretic peptide induces shedding of endothelial glycocalyx in coronary vascular bed of guinea pig hearts. Am J Physiol Heart Circ Physiol. 2005;289:H1993-1999.

18. Dąbrowski W, Kotlińska-Hasiec E, Jaroszyński A, et al. Intra-abdominal pressure correlates with extracellular water content. PLoS One. 2015;10:e0122193. doi: 10.1371/journal.pone.0122193.

19. Navarro LHC, Bloomstone JA, Auler JO Jr, et al. Perioperative fluid therapy: A statement from the International Fluid Optimization Group. Perioper Med. 2015;4:3. doi: 10.1186/s13741-015-0014-z.

20. Ruttmann TG, Montoya-Pelaez LF, James MF. The coagulation changes induced by rapid in vivo crystalloid infusion are attenuated when magnesium is kept at the upper limit of normal. Anesth Analg. 2007;104:1475-1480.

21. Ekseth K, Abildgaard L, Vegfors M, Berg-Johnsen J, Engdahl O. The in vitro effects of crystalloids and colloids on coagulation. Anesthesia. 2002;57:1102-1108.

22. Darlington DN, Delgado AV, Kheirabadi BS, et al. Effect of hemodilution on coagulation and recombinant factor VIla efficacy in human blood in vitro. J Trauma. 2011;71:1152-1163. doi: 10.1097/ TA.0b013e318215178c.

23. Janvrin SB, Davies G, Greenhalgh RM. Postoperative deep vein thrombosis caused by intravenous fluids during surgery. Br J Surg. 1980;67:690-693.

24. Holodinsky JK, Roberts DJ, Ball CG, et al. Risk factors for intraabdominal hypertension and abdominal compartment syndrome among adult intensive care unit patients: A systematic review and meta-analysis. Crit Care. 2013;17:R249. doi: 10.1186/cc13075.

25. Huang SY, Feng CW, Hung HC, et al. A novel zebrafish model to provide mechanistic insights into the inflammatory events in carrageenan-induced abdominal edema. PLoS One. 2014;9:e104414. doi: 10.1371/journal.pone.0104414.

26. Ohmi S, Takei T, Habuka K, Watanabe Y. Acute pulmonary leak syndrome during elective surgery under general anesthesia. J Anesth. 2008;22:77-80.

27. Wakabayashi S, Yamaguchi K, Kumakura S, et al. Effects of anesthesia with sevoflurane and propofol on the cytokine/chemokine production at the airway epithelium during esophagectomy. Int $\mathrm{J} \mathrm{Mol}$ Med. 2014;34:137-144.

28. Rassias AJ, Procopio MA. Stress response and optimization of perioperative care. Dis Mon. 2003;49:522-554.

29. Desborough JP. The stress response to trauma and surgery. Br J Anaesth. 2000;85:109-117.

30. Medrano G, Eitner F, Floege J, Leonhardt S. A novel bioimpedance technique to monitor fluid volume state during hemodialysis treatment. ASAIO J. 2010;56:215-220.

31. Zhu F, Schneditz D, Wang E, Levin NW. Dynamics of segmental extracellular volumes during changes in body position by bioimpedance analysis. J Appl Physiol. 1998;85:497-504.

32. Oshima Y, Shiga T. Within-day variability of whole-body and segmental bioelectrical impedance in a standing position. Eur J Clin Nutr. 2006;60:938-941.

33. Sugano N, Yokoyama K, Kato N, et al. Monitoring of body water composition by the simultaneous use of bioelectrical impedance analysis and Crit-Line" during hemodialysis. Clin Exp Nephrol. 2014;18:944-951. 